\title{
Energy-Efficient Coordinated Transmission for Cloud-RANs: Algorithm Design and Trade-off
}

\author{
Vu Nguyen Ha, Long Bao Le, and Ngọc-Dũng Đào
}

\begin{abstract}
In this paper, we consider the energy-efficient coordinated transmission design for downlink transmission in the cloud radio access network (Cloud-RAN) considering fronthaul capacity and user QoS constraints. Specifically, we assume that baseband signals are processed in the cloud, which are delivered to remote radio heads (RRHs) equipped with multiple antennas over fronthaul links for transmissions to single-antenna users. The design amounts to determine the set of RRHs to serve each user as well as the precoding and power levels for downlink transmission while maintaining the fronthaul capacity and user QoS constraints. Toward this end, we study the two closely-related problems, namely pricing-based total power and fronthaul capacity tradeoff (PFT) and fronthaul-constrained power minimization (FCPM) problems. We employ the concave approximation and gradient search methods to solve the PFT problem for the given pricing coefficients, which capture the power and fronthaul capacity tradeoff. Then, we develop an efficient algorithm to address the FCPM problem by iteratively solving the PFT problem while intelligently updating the pricing coefficients. Numerical results confirm the excellent performance of the our proposed algorithms and illustrate underlying tradeoffs among total transmission power, fronthaul capacity, and cluster size.
\end{abstract}

Index Terms-Cloud radio access network (Cloud-RAN), beamforming, power minimization, resource allocation, clustering.

\section{INTRODUCTION}

The next generation wireless cellular network is expected to provide significant enhancement in capacity to support emerging broadband applications. Coordinated multipoint (CoMP) transmission and reception techniques have been proposed as one of the most important solutions along this line for (LTE)-Advanced systems [1]. For downlink coordinated transmission scheme, several base stations (BSs) cooperate their transmissions to serve each user, which help mitigate intraand inter-cell interference. However, deployment of CoMP in cellular networks typically requires costly high-speed backhauls connecting different BSs for various CSI and information exchanges [2, 3]. Cloud-RAN has been recently proposed as an alternative way to achieve the advantages of CoMP where we exploit the computation power of the cloud to compute and realize most base-band processing functionalities $[4,5]$. Cloud-RAN can also enable to deploy small-cells in existing wireless systems to improve the network coverage and capacity $[6,7]$.

Vu Nguyen Ha and Long Bao Le are with INRS, University of Quebec, Montreal, QC, Canada; e-mails: \{hanguyen,long.le\}@emt.inrs.ca.

Ngọc-Dũng Đào is with Huawei Technologies Canada Co. Ltd., Ottawa, Canada; e-mail: daongocdung@ualberta.net.

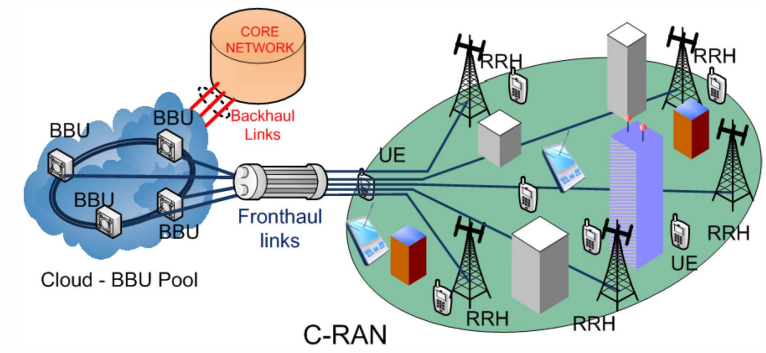

Fig. 1. The Cloud-RAN architecture.

In the Cloud-RAN, various signal processing functions are performed by the digital baseband processing units (BBUs) in the cloud and transmissions of radio signals to users are realized by remote radio heads (RRHs), which form RF signals based on baseband signals received from the cloud. There are various challenges in designing and deploying the CloudRAN architecture. Specifically, we must efficiently utilize the processing resource in the cloud, the fronthaul capacity, and design suitable communication schemes for baseband signal processing. Some of these problems have been studied in recent works. In $[8,9]$, compression techniques was considered to reduce the amount of data transmitted over the backhaul links. The work [10] demonstrated the benefits of Cloud-RAN in the small-cell network.

In this paper, we consider the coordinated transmission problem for downlink power minimization in Cloud-RAN considering practical constraints on the transmission power, users' QoS, and fronthaul capacity. Toward this end, we study two closely related problems, namely "Fronthaul-Constrained Power Minimization" (FCPM) and "Power and Fronthaul Capacity Trade-off" (PFT) problems. In the FCPM problem, we aim to determine the set of RRHs serving each user equipment (UE), and the precoding vectors for RRHs to minimize the total transmission power subject to the constraints on fronthaul capacity. In the PFT problem, the trade-off between transmission power and required fronthaul capacity is optimized via minimizing the weighted sum of total transmission power and fronthaul capacity. In particular, we develop a novel iterative algorithm to determine an efficient solution of the FCPM problem by iteratively solving the PFT problem with adaptively updating pricing coefficients. Moreover, we propose an algorithm to solve the PFT problem which is based on the concave approximation of the fronthaul capacity function and the gradient search method.

The remaining of this paper is organized as follows. We 
describe the system model and problem formulation in Section II. In Section III, we present the proposed algorithms to solve the FCPM and PFT problems. Numerical results are presented in Section IV followed by conclusion in Section V. For notation, we use $\mathbf{X}^{H}, \operatorname{Tr}(\mathbf{X})$ and $\operatorname{rank}(\mathbf{X})$ to denote Hermitian transpose, trace, and rank of matrix $\mathbf{X}$, respectively. $\mathbf{1}_{x \times y}, \mathbf{0}_{x \times y}$ denote the matrix of ones, matrix of zeros whose dimension are $x \times y$, respectively. $|\mathcal{S}|$ denotes the cardinality of set $\mathcal{S}$ and $\operatorname{diag}(\mathrm{x})$ is the diagonal matrix constructed from the elements of vector $\mathrm{x}$.

\section{System MODEL AND PROBLEM Formulation}

\section{A. System Model and Transmission Strategy}

The general architecture of Cloud-RAN is illustrated in Fig. 1, which consist of three main components, namely (i) centralized processors or BBUs pool, (ii) the optical transport network (i.e., fronthaul links), and (iii) remote radio head (RRH) access units with antennas located at the remote sites. The processing center comprising a large number of BBUs is the heart of this architecture where BBUs operate as virtual base stations to process baseband signals for users and optimize the network resource allocation tasks for the system. The transport network connecting the central processing pool and the distributed RRHs is usually deployed using optical fibers. The RRHs transmit RF signals, which are formed by using baseband signals received from BBUs, to UEs in the downlink or forward the baseband signals from UEs to the BBU pool for further processing in the uplink. By conducting most signal processing functions in the cloud (i.e., by BBU pool), RRHs can be relatively simple.

We consider coordinated downlink communications in the Cloud-RAN with $K$ RRHs and $M$ UEs. Let $\mathcal{K}$ and $\mathcal{U}$ be the sets of RRHs and UEs in the network, respectively. The cloud processes baseband signals from which RRHs form RF signals for transmission to the UEs. We assume that each UE is served by a specific group of RRHs, which receive corresponding baseband signals from the cloud. This coordinated transmission scheme aims to exploit antenna resources available at different RRHs to mitigate the interference [1], [2]. Let us denote

$$
t_{u}^{k}= \begin{cases}1 & \text { if RRH } k \text { serves UE } u \\ 0 & \text { otherwise. }\end{cases}
$$

Then the set of RRHs who serve UE $u$ can be determined as

$$
\mathcal{R}_{u}=\left\{k \mid k \in \mathcal{R}, t_{u}^{k}=1\right\} .
$$

For a specific RRH allocation solution represented by $\left\{\mathcal{R}_{1}, \mathcal{R}_{2}, \ldots, \mathcal{R}_{M}\right\}$, the transmission strategy in Cloud-RAN can be summarized as follows. The cloud collects data of UE $u$ from the core network, produces baseband signals, and determine the precoding vectors for the corresponding RRHs. Then, it sends the baseband signals and precoding vectors of each UE $u$ to the serving RRHs in the set $\mathcal{R}_{u}$ over the fronthaul links. Finally, each RRH will up-convert the received baseband signals to the RF band and transmit to corresponding UEs using the received precoding vectors.

In this paper, we consider the MISO transmission from RRHs to UEs where RRH $k$ is equipped with $N_{k}$ antennas and each UE has a single antenna. We assume that UE $u$ receives symbol sequence $x_{u} \in \mathbb{C}$ of unit power, which is transmitted by RRHs in set $\mathcal{R}_{u}$ upon receiving the processed baseband signals from the cloud. Denote $\mathbf{v}_{u}^{k} \in \mathbb{C}^{N_{l} \times 1}$ as the precoding vector at RRH $k$ corresponding to the signal transmitted to UE $u$. Then, the transmission power of the RF signal from RRH $k$ in the set $\mathcal{R}_{u}$ for UE $u$ can be expressed as

$$
p_{u}^{k}=\mathbf{v}_{u}^{k H} \mathbf{v}_{u}^{k}
$$

Let $\mathbf{P}$ be the vector whose elements $p_{u}^{k} \geq 0, \forall k, u$ represents transmission powers $p_{u}^{k}$ given in (3). Specifically, $p_{u}^{k}=0$ implies that RRH $k$ does not serve UE $u$; then, the fronthaul link from the cloud to RRH $k$ does not consume any capacity for carrying the baseband signal of UE $u$. Hence, the transmission power vector $\mathbf{P}$ also indicates the number of baseband signals carried by the fronthaul links, which corresponds to the number of non-zeros elements of $\mathbf{P}$. Moreover, the number of baseband signals carried by the fronthaul link from the cloud to the RRH $k$ can be written as

$$
C_{k}(\mathbf{P})=\sum_{u \in \mathcal{U}} f_{\mathbf{s}}\left(p_{u}^{k}\right)
$$

where $f_{\mathbf{s}}(x)(x \geq 0)$ is the step function which is determined as

$$
f_{\mathbf{s}}(x)= \begin{cases}1 & \text { if } x>0 \\ 0 & \text { if } x=0\end{cases}
$$

We also impose the constraint on the total transmission power of RRH $k$ by its maximum power budget $P_{k}(k \in \mathcal{K})$, which is described as

$$
\sum_{u \in \mathcal{U}} p_{u}^{k} \leq P_{k}, \quad \forall k \in \mathcal{K} .
$$

Given the RRH allocation solution, the corresponding baseband signal $y_{u}$ received at UE $u$ can be written as

$$
y_{u}=\underbrace{\sum_{k \in \mathcal{R}_{u}} \mathbf{h}_{u}^{k H} \mathbf{v}_{u}^{k} x_{u}}_{\text {desired signal }}+\underbrace{\sum_{i=1, \neq u}^{M} \sum_{l \in \mathcal{R}_{i}} \mathbf{h}_{u}^{l H} \mathbf{v}_{i}^{l} x_{i}}_{\text {interference }}+z_{u},
$$

where $\mathbf{h}_{u}^{k} \in \mathbb{C}^{N_{k} \times 1}$ denotes the channel coefficients between RRH $k$ and UE $u$, and $z_{u}$ describes the noise at UE $u$. Also, the signal-to-interference-plus-noise-ratio (SINR) achieved by $\mathrm{UE} u$ is

$$
\Gamma_{u}(\mathbf{V})=\frac{\left|\sum_{k \in \mathcal{R}_{u}} \mathbf{h}_{u}^{k H} \mathbf{v}_{u}^{k}\right|^{2}}{\sum_{i=1, \neq u}^{M}\left|\sum_{l \in \mathcal{R}_{i}} \mathbf{h}_{u}^{l H} \mathbf{v}_{i}^{l}\right|^{2}+\sigma^{2}}
$$

where $\sigma^{2}$ is the noise power and $\mathbf{V}$ is the vector formed by concatenating elementary vectors $\mathbf{v}_{u}^{k}, k \in \mathcal{R}_{u}$, which represent the corresponding precoding vector.

We further assume that each UE $u$ aims to maintain her required QoS which is represented by the corresponding target SINR $\bar{\gamma}_{u}$. Then, this QoS requirement can be described as

$$
\Gamma_{u}(\mathbf{V}) \geq \bar{\gamma}_{u}, \quad \forall u \in \mathcal{U}
$$




\section{B. RRH Clustering Constraints}

To limit the computational complexity in large networks, we can limit that each UE may only be served by certain nearby RRHs. Denote $\mathcal{K}_{u}\left(\mathcal{K}_{u} \subseteq \mathcal{K}\right)$ as the maximum set of RRHs which are allowed to serve UE $u$. Note, however, that the set of RRHs $\mathcal{R}_{u}$ due to our proposed algorithm would be one sub-set of $\mathcal{K}_{u}$. Accordingly, we can also define the set of UEs which can be served by RRH $k$ as

$$
\mathcal{U}_{k}=\left\{u \mid u \in \mathcal{U}, k \in \mathcal{K}_{u}\right\}
$$

Under these clustering constraints, RRH $k$ can only receive from the cloud the baseband signals of UEs in set $\mathcal{U}_{k}$. Accordingly, the utilized fronthaul capacity $C_{k}$ is upper bounded by both the cardinality of $\mathcal{U}_{k}$ and the available fronthaul capacity. We employ $C_{k}^{\max }=\left|\mathcal{U}_{k}\right|$ to denote this maximum limit in this paper.

Remark 1. If the UEs are allowed to communicate with all RRHs in the network then we have $\mathcal{K}_{u}=\mathcal{K}$ for all $u \in \mathcal{U}$; therefore, $\mathcal{U}_{k}=\mathcal{U}$ and $C_{k}^{\max }=M$ for all $k \in \mathcal{K}$.

\section{Problem Formulation}

In this following, we present the formulations for the FCPM and PFT problems. These problems aim to determine the set of RRHs serving each UE and the corresponding precoding vectors for all RRHs (i.e., represented by $\mathbf{V}$ ) to minimize the total transmission power under the constrained fronthaul capacity in the FCPM problem, and to minimize the weighted sum of total transmission power and fronthaul capacity in the PFT problem.

1) Fronthaul-Constrained Power Minimization Problem: In this problem, we assume that the fronthaul link between the cloud and RRH $k$ is capable of carrying at most $\bar{C}_{k}$ baseband signals for UEs. This is transferred in the following fronthaul capacity constraints

$$
C_{k}(\mathbf{P}) \leq \bar{C}_{k}, \quad \forall k \in \mathcal{K}
$$

where we have $\bar{C}_{k} \leq C_{k}^{\max }, \forall k \in \mathcal{K}$ due to the clustering constraints described above. We are now ready to state the FCPM problem as

$$
\begin{aligned}
& \min _{\mathbf{V}, \mathbf{P}} \sum_{k \in \mathcal{K}} \sum_{u \in \mathcal{U}} p_{u}^{k} \\
& \text { s.t. constraints (3), (6), (9), (11). }
\end{aligned}
$$

The optimization problem (12) is indeed a mixed integer program, which is an NP-hard problem.

2) Power and Fronthaul Capacity Trade-off Problem: We start by introducing a pricing coefficient $q_{k}$ for RRH $k$, which can be interpreted as the price per unit fronthaul capacity for the link between the cloud and RRH $k$. Then, the PFT problem can be formulated as

$$
\begin{aligned}
& \min _{\mathbf{V}, \mathbf{P}} \sum_{k \in \mathcal{K}} \sum_{u \in \mathcal{U}} p_{u}^{k}+\sum_{k \in \mathcal{K}} q_{k} C_{k}(\mathbf{P}) \\
& \text { s.t. } \quad \text { constraints (3), (6), (9). }
\end{aligned}
$$

3) Relationship between FCPM and PFT Problems: Let $\overline{\mathbf{C}}_{\mathrm{FCPM}}=\left[\bar{C}_{1}, \bar{C}_{2}, \ldots, \bar{C}_{K}\right]$ denote the vector of maximum fronthaul capacity limits and $\mathbf{C}_{\mathrm{PFT}}^{\mathrm{opt}}(\mathbf{q})=$ $\left[C_{1}^{\text {opt }}(\mathbf{q}), C_{2}^{\text {opt }}(\mathbf{q}), \ldots, C_{K}^{\text {opt }}(\mathbf{q})\right]$ describes the vector of optimum fronthaul link capacity due to the PFT problem for a given price coefficient vector $\mathbf{q}=\left[q_{1}, q_{2}, \ldots, q_{K}\right]$. Then, it can be verified that the optimal precoding vectors and the transmission powers of FCPM and PFT problems are the same if $\mathbf{C}_{\mathrm{PFT}}^{\mathrm{opt}}(\mathbf{q})=\overline{\mathbf{C}}_{\mathrm{FCPM}}$. This important fact enables us to develop an adaptive algorithm to solve the FCPM problem based on adjusting the pricing parameters $q$ and solving PFT problem iteratively in attempting to attain $\mathbf{C}_{\mathrm{PFT}}^{\mathrm{opt}}(\mathbf{q})=\overline{\mathbf{C}}_{\mathrm{FCPM}}$.

\section{AlgorithM DESIGN}

We describe our proposed algorithm to solve the FCPM problem based on the solution of the PFT problem first. Then, we present the algorithm design to address the PFT problem.

\section{A. FCPM Problem Solution}

We propose a novel algorithm to solve the FCPM problem by iteratively solving the PFT problem while smartly adjusting the pricing coefficients $\left\{q_{k}\right\}$. Our proposed algorithm is developed based on assumption of that the PFT problem can be solved, which will be described in the next subsection. The key idea is to adjust the pricing PFT problem so that $\mathbf{C}_{\mathrm{PFT}}^{\mathrm{opt}}(\mathbf{q})$ is equal to $\overline{\mathbf{C}}_{\mathrm{FCPM}}$. This algorithm is explained in the following.

At the first iteration, $\mathbf{q}$ is set so that $\mathbf{C}_{\mathrm{PFT}}^{\mathrm{opt}}(\mathbf{q})$ is as large as possible. This can be achieved by setting $\mathbf{q}=\mathbf{0}_{K \times 1}$, which translates the PFT problem into the power minimization problem (PM) without any fronthaul constraint. Since the bigger set of $\left\{\mathcal{R}_{u}\right\}$ provides larger feasible set of $\mathbf{V}$, the optimum solution of PM can be achieved as $\mathcal{R}_{u}=\mathcal{K}_{u}$. This implies that we have $C_{k}^{\mathrm{opt}}(\mathbf{q})=C_{k}^{\max }$ for all $k \in \mathcal{K}$. Then, we iteratively adjust the pricing coefficients to decrease elements in $\mathbf{C}_{\mathrm{PFT}}^{\mathrm{opt}}(\mathbf{q})$ toward their maximum allowable limits $\overline{\mathbf{C}}_{\mathrm{FCPM}}$. In the following, we establish some theoretical results based on which we develop the mechanisms to update the pricing coefficients.

Proposition 1. Suppose that $q_{i}$ are fixed for all RRHs $i \in \mathcal{K} / k$ then we have

1) $C_{k}^{\mathrm{opt}}(\mathbf{q})$ is a decreasing function of $q_{k}$.

2) There exists an upper bound $\delta_{k}$ for RRH $k$ where if $q_{k} \geq$ $\delta_{k}$ then $C_{k}^{\mathrm{opt}}(\mathbf{q})$ can not be further decreased.

3) When $q_{k} \geq \delta_{k}$, if $C_{k}^{\mathrm{opt}}(\mathbf{q})>\bar{C}_{k}, F C P M$ is infeasible.

Proof. The proof is given in Appendix A.

Proposition 2. For any $\mathbf{q}$, if we have $\sum_{k \in \mathcal{K}} q_{k}\left(C_{k}^{\mathrm{opt}}(\mathbf{q})-\right.$ $\left.\bar{C}_{k}\right) \geq \sum_{k \in \mathcal{K}} P_{k}$, then FCPM problem is infeasible.

Proof. The proof can be found in Appendix B.

These results form the foundation based on which we can develop an iterative algorithm presented in Algorithm 1, which describes how the the pricing coefficients are updated over iterations. In the $l^{\text {th }}$ iteration, the main operation is conducted in steps 7-8 where other steps establish the stopping conditions based on the results in Propositions 1 and 2 . In step 8, we 


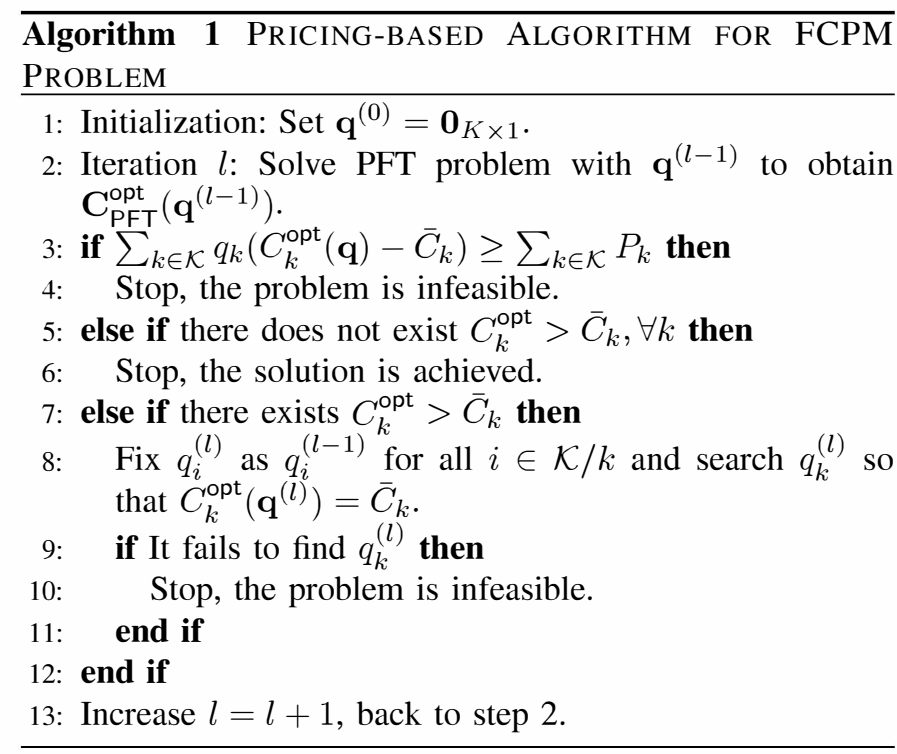

attempt to decrease the value of $C_{k}^{\mathrm{opt}}(\mathbf{q})$ which was greater than the allowable limit $\bar{C}_{k}$ by searching the value of $q_{k}^{(l)}$ in $\left(q_{k}^{(l-1)}, \delta_{k}^{(l-1)}\right)$ in order to decrease $C_{k}^{\text {opt }}(\mathbf{q})$ toward $\bar{C}_{k}$. The binary searching method can be employed. If it fails to find such $q_{k}^{(l)}$, FCPM is infeasible according the third statement of Proposition 1. The algorithm will stop if $C^{\text {opt }}$ converges to $\overline{\mathbf{C}}$. Otherwise, one element $q_{k}$ of $\mathbf{q}$, whose the corresponding $C_{k}^{\text {opt }}(\mathbf{q})$ is greater than $\bar{C}_{k}$, is increased in each iteration as described in Algorithm 1. In any particular iteration $\left(l_{*}\right)$, if we have $\sum_{k \in \mathcal{K}} q_{k}^{\left(l_{*}\right)}\left(C_{k}^{\text {opt }}\left(\mathbf{q}^{\left(l_{*}\right)}\right)-\bar{C}_{k}\right) \geq \sum_{k \in \mathcal{K}} P_{k}$ and $C_{k}^{\mathrm{opt}}\left(\mathbf{q}^{l^{*}}\right) \geq \bar{C}_{k}(\forall k)$, we can conclude that the problem FCPM is infeasible by invoking the results in Proposition 2.

\section{B. PFT Problem Solution}

We now develop an efficient algorithm to solve the PFT problem based on concave approximation of the function $f_{\mathbf{s}}(\cdot)$. For $x \geq 0$, the step function $f_{\mathrm{s}}(x)$ can be approximated to the concave penalty function $f_{\text {exp }, \Psi}(x)$ as

$$
f_{\mathbf{s}}(x) \approx 1-e^{-\Psi x},
$$

where $\Psi \gg 1$. Using this approximation, the PFT problem can be relaxed to the following problem

$$
\begin{aligned}
& \min _{\mathbf{P}, \mathbf{V}} \sum_{k \in \mathcal{K}} \sum_{u \in \mathcal{U}} p_{u}^{k}+\sum_{k \in \mathcal{K}} \sum_{u \in \mathcal{U}} q_{k}\left(1-e^{-\Psi p_{u}^{k}}\right) \\
& \text { s.t. constraints (3), (6), (9) }
\end{aligned}
$$

where the objective function is concave and the feasible region corresponding to all the constraints is convex. This problem is of the following general form

$$
\min _{\mathbf{x}} g(\mathbf{x}) \text { subject to } \mathbf{x} \in \mathcal{F},
$$

where $g(\mathbf{x})$ is concave with respect to $\mathbf{x}$ and $\mathcal{F}$ is the corresponding feasible region. This problem can be solved by using the gradient method as follows. We start with $\mathrm{x}^{(0)} \in \mathcal{F}$ and then iteratively determine the better solution as

$$
\mathbf{x}^{(n+1)}=\arg \min _{\mathbf{x}} g\left(\mathbf{x}^{(n)}\right)+\nabla g\left(\mathbf{x}^{(n)}\right)\left(\mathbf{x}-\mathbf{x}^{(n)}\right) \text { s.t. } \mathbf{x} \in \mathcal{F} \text {. }
$$

In particular, $\mathbf{x}^{(n+1)}$ needs to be determined from the following problem

$$
\min _{\mathbf{x}} \nabla g\left(\mathbf{x}^{(n)}\right) \mathbf{x} \text { s.t. } \mathbf{x} \in \mathcal{F} \text {. }
$$

We now go back to our problem where we let $\mathrm{x}$ represent to all precoding vectors and powers, and $g(\mathbf{x})$ denote the objective function of the problem (15). Then, we have $\nabla g(\mathbf{x}) \mathbf{x}=\sum_{\forall(u, k)}\left(1+q_{k} \Psi e^{-\Psi p_{u}^{k}}\right) p_{u}^{k}$. By applying the gradient method, we can solve problem (15) by iteratively by solving the following problem until the convergence

$$
\begin{aligned}
& \min _{\mathbf{V}} \sum_{k \in \mathcal{K}} \sum_{u \in \mathcal{U}} \alpha_{u}^{k(n)} \mathbf{v}_{u}^{k H} \mathbf{v}_{u}^{k} \\
& \text { s.t. } \quad \text { constraints (6), (9). }
\end{aligned}
$$

where

$$
\alpha_{u}^{k(n)}=1+q_{k} \Psi e^{-\Psi p_{u}^{k,(n-1)}} .
$$

The problem (19) is a weighted sum-power minimization problem, which can be transformed into the well-known convex semi-definite program (SDP) as follows.

1) Weighted Sum-Power Minimization Solution: For the predetermined sets $\left\{\mathcal{K}_{u}\right\}$, let $\mathbf{v}_{u}$ denote the precoding solution over all RRHs in set $\mathcal{K}_{u}$ serving user $u$, which is defined as $\mathbf{v}_{u}=\left[\mathbf{v}_{u}^{u_{1}}, \mathbf{v}_{u}^{u_{2}} \ldots \mathbf{v}_{u}^{u_{u} u}\right]$ where $\left\{u_{1}, \ldots, u_{a_{u}}\right\}=\mathcal{K}_{u}$ and $a_{u}=$ $\left|\mathcal{K}_{u}\right|$. So, we have $\mathbf{v}_{u} \in \mathbb{C}^{N_{u} \times 1}$ where $N_{u}=\sum_{k \in \mathcal{K}_{u}} N_{k}$. Here, we are interested in determining $\mathbf{v}_{u}$ because we have $\mathbf{v}_{u}^{i}=\mathbf{0}$ for all $i \notin \mathcal{K}_{u}$ (i.e., the precoding vector is equal to zero for any RRH that does not serve the underlying UE).

Let us define $\mathbf{W}_{u}=\mathbf{v}_{u} \mathbf{v}_{u}^{H}$, we have $\mathbf{W}_{u} \in \mathbb{C}^{N_{u} \times N_{u}}$. It is positive semi-definite $\left(\mathbf{W}_{u} \succeq \mathbf{0}\right)$ and has rank one because it is generated from vector $\mathbf{v}_{u}$. We also define the channel vector $\mathbf{h}_{u}^{i}=\left[\mathbf{h}_{u}^{i_{1}}, \mathbf{h}_{u}^{i_{2}}, \ldots \mathbf{h}_{u}^{i_{\boldsymbol{e}_{i}}}\right]$ and $\mathbf{H}_{u}^{i}=\mathbf{h}_{u}^{i} \mathbf{h}_{u}^{i H}$ for all $u, i \in \mathcal{U}$. Then, the SINR constraint for UE $u$ in (9), and the power constraint for RRHs can be rewritten in the matrix forms as

$$
\begin{array}{r}
\operatorname{Tr}\left(\mathbf{H}_{u}^{u} \mathbf{W}_{u}\right)-\bar{\gamma}_{u} \sum_{i \in \mathcal{U} / u} \operatorname{Tr}\left(\mathbf{H}_{u}^{i} \mathbf{W}_{i}\right) \geq \bar{\gamma}_{u} \sigma^{2}, \quad \forall u \in \mathcal{U}, \\
\sum_{u \in \mathcal{U}} \operatorname{Tr}\left(\mathbf{E}_{u}^{k} \mathbf{W}_{u}\right) \leq P_{k}, \quad \forall k \in \mathcal{K}
\end{array}
$$

where $\mathbf{E}_{u}^{k}=\operatorname{diag}\left(\mathbf{0}_{N_{u_{1}} \times 1}, \ldots, \mathbf{1}_{N_{u_{i}} \times 1}, \ldots, \mathbf{0}_{N_{u_{\bullet_{u}}} \times 1}\right)$ if $u_{i}=$ $k$. Therefore, the weighted sum-power minimization can be formulated as the following SDP problem

$$
\begin{aligned}
& \min _{\left\{\mathbf{W}_{u}\right\}_{u=1}^{M}} \sum_{u=1}^{M} \operatorname{Tr}\left(\mathbf{D}_{u} \mathbf{W}_{u}\right) \\
& \text { s.t. } \quad \text { constraints }(21),(22), \\
& \quad \mathbf{W}_{u} \succeq \mathbf{0}, \operatorname{rank}\left(\mathbf{W}_{u}\right)=1, \forall u
\end{aligned}
$$

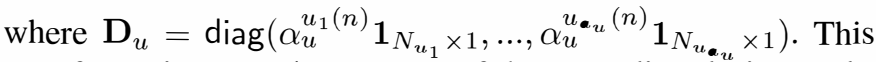
transformation reveals structure of the precoding design problem. Specifically, if we remove the rank-one constraints $\operatorname{rank}\left(\mathbf{W}_{u}\right)=1$ from (23) then the resulting problem is convex. In fact, this relaxed problem is the semi-definite program (SDP), which is convex and therefore can be solved easily by using standard tools such as CVX solver [11]. As given in Theorem 3.1 of [12], if (23) is feasible, then it has at least one solution where $\operatorname{rank}\left(\mathbf{W}_{u}\right)=1$, for all $u \in \mathcal{U}$. Then, $\mathbf{v}_{u}$ will be calculated as the eigenvector of $\mathbf{W}_{u}$. 


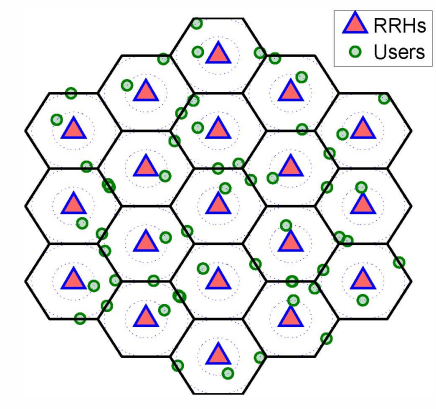

Fig. 2. Simulation model

2) Algorithm Design: The algorithm to solve (15) is given in Algorithm 2. The convergence of Algorithm 2 follows from the convergence guarantee of the gradient method (18).

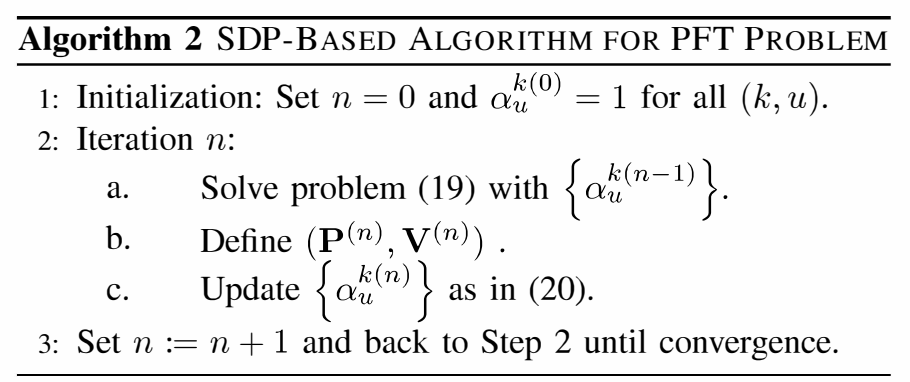

\section{Design Summary}

Algorithm 1, which is proposed to solve the FCPM problem, is based on the solution of the PFT problem, which can be obtained by using Algorithm 2. Note that the maximum cluster size will determine the computational complexity of our design since the size of the precoding optimization variables in (19) and (23) will vary accordingly.

\section{NumericAl Results}

The effectiveness of our proposed design is validated through simulations based on the 19-cell $(\mathrm{K}=19)$ wrap-around network setup with 3 users per cell $(M=57)$ and 4 transmit antennas $\left(N_{k}=4\right)$ at each RRH as illustrated in Fig. 2. The UEs are randomly located inside each cell so that distance between them and their nearest RRH is $d$ or $d / 2$. The distance between two nearest RRHs are $2 d$ where $d=250 \mathrm{~m}$. The channel gains are generated by considering both Rayleigh fading and path loss which is modeled as $L_{u}^{k}=36.8 \log _{10}\left(d_{u}^{k}\right)+43.8+20 \log _{10}\left(\frac{f_{c}}{5}\right)$ where $d_{u}^{k}$ is the distance from UE $k$ to RRH $k ; f_{c}=2.5 \mathrm{GH} z$. The noise power is set equal to $\sigma^{2}=10^{-13} \mathrm{~W} . \Psi=10^{3}$ and $P_{k}=10 \mathrm{~W}$ for all $k \in \mathcal{K}$.

In Fig. 3, we present the variations of total transmission power of all RRHs versus the total capacity of all fronthaul links. To obtain these results, we set all pricing coeffcients equal to $q$ and vary this value to achieve the trade-off curve between transmission power and total fronthaul capacity. As can be observed, the transmission power decreases if we have larger fronthaul capacity. However, the power becomes

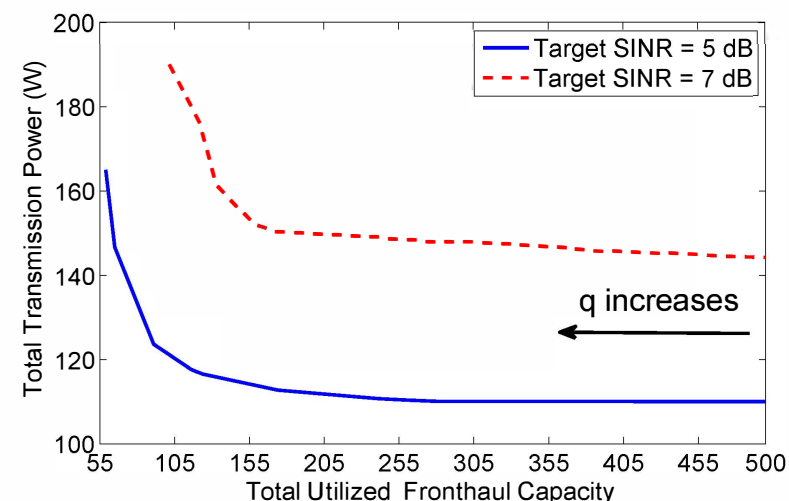

Fig. 3. Total transmission power versus the total fronthaul capacity.

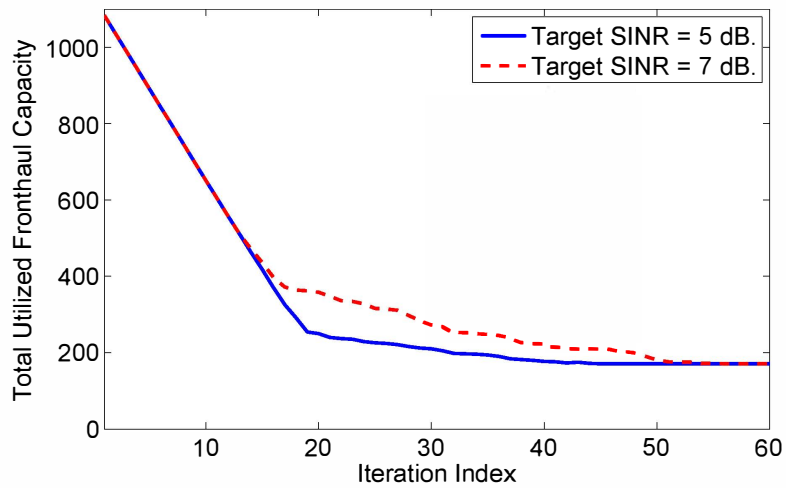

Fig. 4. Total fronthaul capacity versus the iteration index.

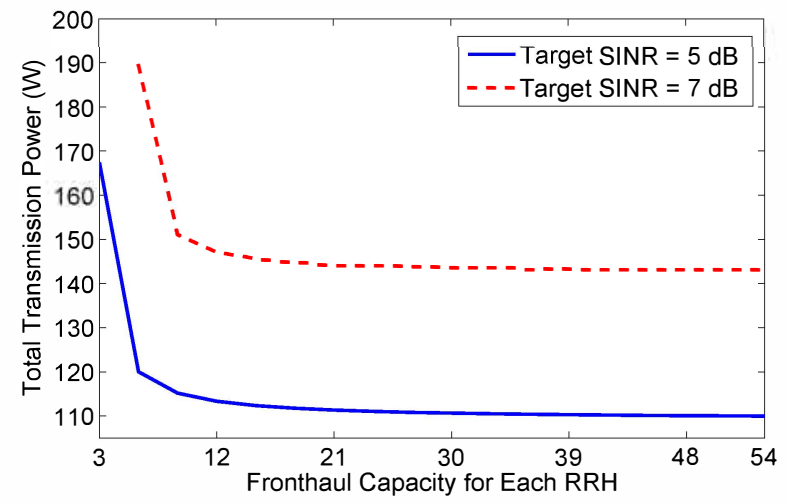

Fig. 5. Total transmission power versus the fronthaul capacity.

saturated as the total fronthaul capacity is sufficiently large. This tradeoff illustrates the design intuition where we can utilize these tradeoff results to determine the operating point of the FCPM problem.

Fig. 4 shows variations of the utilized fronthaul capacity over iterations due to Algorithm 1. This confirms the convergence of our proposed algorithm. In addition, the higher target SINRs result in longer convergence time. Finally, Figs. 5 and 6 illustrate how the total transmission power varies with the fronthaul capacity and the cluster size (i.e., maximum number 


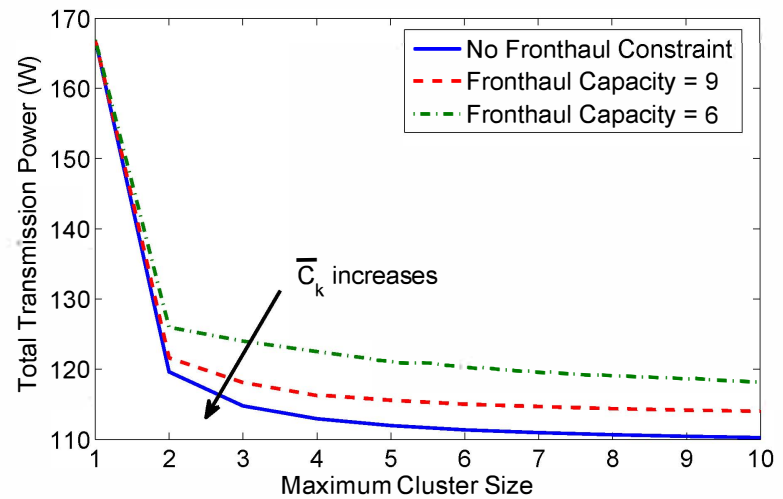

Fig. 6. Total transmission power versus maximum cluster size (maximum number of RRHs serving one user).

of RRHs serving one user), respectively. As can be seen, the network can achieve the better performance when the fronthaul capacity is larger as shown in Fig. 5. Also, the larger cluster size results in smaller total transmission power as confirmed by Fig. 6. Recall, however, that larger cluster size increases the computational complexity of our proposed algorithms.

\section{CONCLUSION}

We have presented efficient and low-complexity algorithms for downlink coordinated transmission in Cloud-RAN where we aim to minimize the total transmission power subject to constraints on transmission powers, fronthaul capacity, and required QoS of users. Numerical results have illustrated the efficacy of our proposed algorithms and the impacts of different parameters on the network performance.

\section{APPENDIX A}

\section{PROOF OF PROPOSITION 1}

\section{A. Proof of Statement 1 in Proposition 1}

We consider two pricing parameters vectors $\mathbf{q}$ and $\mathbf{q}^{\prime}$ where $q_{k}^{\prime}>q_{k}$ and $q_{i}^{\prime}=q_{i}$ for all $i \neq k$. Let $(\mathbf{P}, \mathbf{V})$ and $\left(\mathbf{P}^{\prime}, \mathbf{V}^{\prime}\right)$ be the solutions of PFT problem with $\mathbf{q}$ and $\mathbf{q}^{\prime}$, respectively. Then, we have

$$
\begin{aligned}
& \sum_{\forall(u, j)} p_{u}^{j}+\sum_{j \in \mathcal{K}} q_{j} C_{j}^{\mathrm{opt}}(\mathbf{q}) \leq \sum_{\forall(u, j)} p_{u}^{j \prime}+\sum_{j \in \mathcal{K}} q_{j} C_{j}^{\mathrm{opt}}\left(\mathbf{q}^{\prime}\right) \\
& \sum_{\forall(u, j)} p_{u}^{j \prime}+\sum_{j \in \mathcal{K}} q_{j}^{\prime} C_{j}^{\mathrm{opt}}\left(\mathbf{q}^{\prime}\right) \leq \sum_{\forall(u, j)} p_{u}^{j}+\sum_{j \in \mathcal{K}} q_{j}^{\prime} C_{j}^{\mathrm{opt}}(\mathbf{q})
\end{aligned}
$$

After combining and simplifying these two inequalities, we have $\left(q_{k}^{\prime}-q_{k}\right) C_{k}^{\text {opt }}\left(\mathbf{q}^{\prime}\right) \leq\left(q_{k}^{\prime}-q_{k}\right) C_{k}^{\text {opt }}(\mathbf{q})$. Then, we have finished the proof for the first statement.

\section{B. Proof of Statement 2 in Proposition 1}

Let $P^{\mathrm{t}}=\sum_{k \in \mathcal{K}} P_{k}$. We choose $\delta_{k}=P^{\mathrm{t}}+\sum_{i \neq k} q_{i} C_{i}^{\text {max }}$. Now, we prove that for any $(\mathbf{P}, \mathbf{V})$ satisfying constraints (3), (6), (9), we all have $C_{k}(\mathbf{P}) \geq C_{k}^{\mathrm{opt}}(\mathbf{q})$ if $q_{k} \geq \delta_{k}$. Let $P^{\mathrm{opt}}(\mathbf{q})$ be the total power solution of PFT problem with q. Because $(\mathbf{P}, \mathbf{V})$ satisfying constraints (3), (6), (9) we have

$$
\begin{aligned}
& P^{\mathrm{opt}}(\mathbf{q})+\sum_{i \in \mathcal{K}} q_{i} C_{i}^{\mathrm{opt}}(\mathbf{q}) \leq \sum_{(u, k)} p_{u}^{k}+\sum_{i \in \mathcal{K}} q_{i} C_{i}(\mathbf{P}) \\
\rightarrow & C_{k}^{\mathrm{opt}}(\mathbf{q})-C_{k}(\mathbf{P}) \leq \frac{P^{\mathrm{opt}}(\mathbf{q})-\sum_{(u, k)} p_{u}^{k}+\sum_{i \neq k} q_{i} C_{i}^{\mathrm{opt}}(\mathbf{q})-C_{i}(\mathbf{P})}{q_{k}}
\end{aligned}
$$

Then, we have $C_{k}^{\text {opt }}(\mathbf{q})-C_{k}(\mathbf{P})<1$ if $q_{k} \geq \delta_{k}$. In addition, $C_{k}^{\text {opt }}(\mathbf{q})$ and $C_{k}(\mathbf{P})$ are integers. Hence, $C_{k}(\mathbf{P})$ cannot be smaller than $C_{k}^{\mathrm{opt}}(\mathbf{q})$.

\section{Proof of Statement 3 in Proposition 1}

From the proof of statement 2, we can see that there exists no $(\mathbf{P}, \mathbf{V})$ satisfying constraints (3), (6), (9) so that $C_{k}(\mathbf{P})=$ $\bar{C}_{k}<C_{k}^{\text {opt }}(\mathbf{q})$. Hence, the last statement of Proposition 1 is proved.

\section{APPENDIX B}

\section{PROOF OF PROPOSITION 2}

We assume that there is $\mathbf{q}$ so that $\sum_{k \in \mathcal{K}} q_{k}\left(C_{k}^{\text {opt }}(\mathbf{q})-\bar{C}_{k}\right) \geq$ $\sum_{k \in \mathcal{K}} P_{k}$. If the problem FCPM is feasible, then there exists $(\mathbf{P}, \mathbf{V})$ satisfying constraints (3), (6), (9) so that $C_{k}(\mathbf{P})=\bar{C}_{k}$ for all $k \in \mathcal{K}$. Then, we have

$$
\begin{array}{r}
P^{\mathrm{opt}}(\mathbf{q})+\sum_{i \in \mathcal{K}} q_{k} C_{k}^{\mathrm{opt}}(\mathbf{q}) \leq \sum_{(u, k)} p_{u}^{k}+\sum_{i \in \mathcal{K}} q_{k} \bar{C}_{k} \\
\sum_{k \in \mathcal{K}} q_{k}\left(C_{k}^{\mathrm{opt}}(\mathbf{q})-\bar{C}_{k}\right) \leq \sum_{(u, k)} p_{u}^{k}-P^{\mathrm{opt}}(\mathbf{q})<\sum_{k \in \mathcal{K}} P_{k}
\end{array}
$$

This results in a contradiction. Hence, the problem FCPM must be infeasible.

\section{REFERENCES}

[1] M. Sawahashi, Y. Kishiyama, A. Morimoto, D. Nishikawa, and M. Tanno, "Coordinated multipoint transmission/reception techniques for LTE-advanced [Coordinated and distributed MIMO]," IEEE Wireless Commun., vol. 17, no. 3, pp. 26-34, Jun. 2010.

[2] R. Irmer, H. Droste, P. Marsch, M. Grieger, G. Fettweis, S. Brueck, H.P Mayer, L. Thiele, and V. Jungnickel, "Coordinated multipoint Concepts, performance, and field trial results," IEEE Commun. Mag., vol. 49, no. 2, pp. 102-111, Feb. 2011.

[3] P. Marsch and G. Fettweis, "Uplink CoMP under a constrained backhaul and imperfect channel knowledge," IEEE Trans. Wireless Commun., vol. 10 , no. 6 , pp. $1730-1742$, Jun. 2011.

[4] "C-RAN: The road towards green ran," White Paper, China Mobile, 2011.

[5] "Suggestion on potential solution to C-Ran," NGMN alliance, Jan. 2013

[6] N. Saquib, E. Hossain, Long B. Le, and D. I. Kim, "Interference management in OFDMA femtocell networks: Issues and approaches," IEEE Wireless Commun., vol. 19, no. 3, pp. 86-95, Jun. 2012.

[7] $\mathrm{Vu} \mathrm{N}$. Ha and Long B. Le, "Distributed base station association and power control for heterogeneous cellular networks," IEEE Trans. Veh. Technol., Jan. 2014.

[8] S.-H. Park, O. Simeone, O. Sahin and S. Shamai (Shitz), "Robust and efficient distributed compression for cloud radio access networks," IEEE Trans. Veh. Technol., vol. 62, no. 2, pp. 692-703, Feb. 2013.

[9] S.-H. Park, O. Simeone, O. Sahin and S. Shamai (Shitz), "Robust layered transmission and compression for distributed uplink reception in cloud radio access networks," IEEE Trans. Veh. Technol., to appear.

[10] C. Liu, K. Sundaresan, M. Jiang, S. Rangarajan, and G.-K. Chang, "The case for re-configurable backhaul in cloud-RAN based small cell networks," in Proc. IEEE INFOCOM, 2013.

[11] M. Grant, S. Boyd, and Y. Ye, "CVX: Matlab software for disciplined convex programming," 2009. Online: http://www.stanford.edu/boyd/cvX.

[12] M. Bengtsson and B. Ottersten, "Optimal downlink beamforming using semidefinite optimization," in Proc. Annual Allerton Conf. on Commun., Control and Computing, Monticello, NY, pp. 987-996, Sept. 1999. 